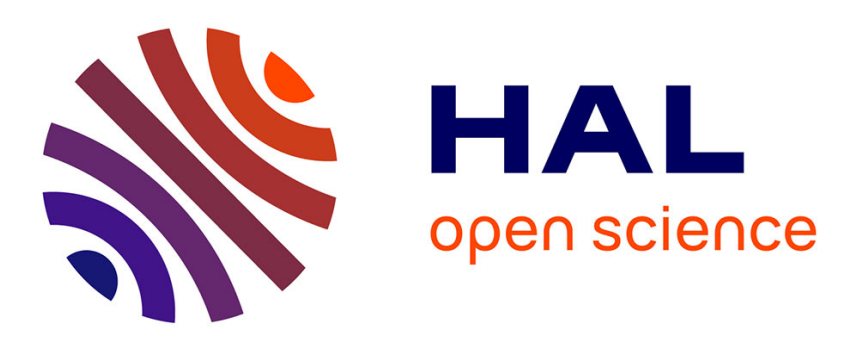

\title{
Scheduling in Massive MIMO: User Clustering and Pilot Assignment
}

\author{
Salah Eddine Hajri, Mohamad Assaad, Giuseppe Caire
}

\section{To cite this version:}

Salah Eddine Hajri, Mohamad Assaad, Giuseppe Caire. Scheduling in Massive MIMO: User Clustering and Pilot Assignment. 54th Annual Allerton Conference on Communication, Control, and Computing, Sep 2016, Illinois, United States. 10.1109/allerton.2016.7852217 . hal-01383769

\section{HAL Id: hal-01383769 https://hal.science/hal-01383769}

Submitted on 27 Oct 2016

HAL is a multi-disciplinary open access archive for the deposit and dissemination of scientific research documents, whether they are published or not. The documents may come from teaching and research institutions in France or abroad, or from public or private research centers.
L'archive ouverte pluridisciplinaire HAL, est destinée au dépôt et à la diffusion de documents scientifiques de niveau recherche, publiés ou non, émanant des établissements d'enseignement et de recherche français ou étrangers, des laboratoires publics ou privés. 


\title{
Scheduling in Massive MIMO: User Clustering and Pilot Assignment
}

\author{
Salah Eddine Hajri*, Student IEEE, Mohamad Assaad*, Senior IEEE, and Giuseppe Caire ${ }^{\dagger}$, Fellow IEEE \\ *Laboratoire des Signaux et Systemes (L2S, CNRS), CentraleSupelec, Gif-sur-Yvette, France \\ $\dagger$ Technische Universität Berlin, Chair of Communications and Information Theory \\ Einsteinufer 25, 10587 Berlin, Germany \\ Email: \{Salaheddine.hajri, Mohamad.Assaad\}@ centralesupelec.fr \\ Caire@tu-berlin.de
}

\begin{abstract}
In this paper, we consider the problem of user scheduling and pilot assignment in TDD multicell multiuser Massive MIMO systems. While in TDD systems the channel is acquired using uplink pilots, we propose a scheme that utilizes additional downlink probing in order to improve the spectral efficiency. The idea is to dynamically assign mobile users to different clusters based on the directions of their channels through the use of downlink reference beams. This will result in forcing the interference to be centered in semiorthogonal subspaces without the need for important feedback and therefore enabling reduction of the pilot contamination effect. The scheduled users in each cluster employs orthogonal training sequences with a pilot reuse factor of 1 among the clusters. We then propose a graphical framework for pilot assignment. We show that this problem can be modeled as a max cut problem and we provide an approximation algorithm that optimizes the pilot allocation.
\end{abstract}

\section{INTRODUCTION}

Multiuser MIMO is one of the main technologies that has been adopted for wireless networks. It enables serving users in the same frequency band and time, exploiting the degrees of freedom in the spatial domain. Coupled with a large antenna array at the Base Station, this system enables a huge increase in the network spectral and energy efficiency. Massive MIMO was first proposed in [1]. The idea was to mimics spreadspectrum used in $3 \mathrm{G}$ networks in which a large processing gain can be realized by massive use of radio spectrum. This gain is imitated through the use of a large number of antennas. Massive MIMO systems were intensively investigated and shown to have a huge potential in improving the spectral and energy efficiency of wireless networks. In [1], the author showed that Massive MIMO systems require channel state information in order to enable simple linear beamforming. It was shown that the spectral efficiency of the massive MIMO system is limited by the CSI imperfection due to pilot contamination. With massive MIMO, the impact of uncorrelated receiver noise, fast fading and interference is decreasing as the number of antennas increases. Usually, when using pilot aided channel estimation, a limiting factor arises since we are required to reuse the same orthogonal pilot sequences in neighboring

The research of M. Assaad has been partially funded by Huawei, France and "Fondation Supelec" cells. This results in pilot contamination. This phenomenon was heavily investigated and many works proposed different methods to deal with it. In [2], the authors proposed a Coordinated Approach to Channel Estimation, in which exploitation of covariance information under certain conditions on the covariance matrices can lead to a complete removal of pilot contamination effects in the large antenna limit. In [3], Joint Spatial Division and Multiplexing (JSDM) for multiuser MIMO downlink was investigated. JSDM is a scheme that aims to serve users by clustering them into groups such that users within a group have approximately similar channel covariances, while users across groups have near orthogonal covariances. In [4], a two-stage beamforming method for massive MIMO broadcast channel in FDD mode was proposed. The key ideas was to use a set of reference beams in order to select semi-orthogonal users and then apply zero-forcing beamforming using the CSI feedback from the selected users. In our paper, we revisit user scheduling for Massive MIMO, based on the channel covariance informations. We consider an a scheduling method where users are grouped according to their channel directions. We concentrate on time division duplexing (TDD) systems, in which channel reciprocity can be used to get estimates of the downlink channels through uplink training, thereby eliminating the need for feedback. This has the important advantage of enabling the usage of the full bandwidth efficiently for data transmission compared with the FDD case where a portion of the available bandwidth is reserved for feedback. We consider a multicell system and we aim at reducing the pilot contamination effect, resulting in an improved spectral efficiency. The proposed scheme consists of using random beamforming [6] for downlink probing in TDD mode. This enables to schedule users in different clusters according to their channel directions. The proposed scheme allows to reduce the feedback to the index of the subspace on which users' channel is concentrated. The users in each cluster will then employ orthogonal training sequences in the uplink and the same set of pilots are reused in all clusters within a given cell. This allows more aggressive pilot reuse. While this scheme allows improvement in the spectral efficiency, it cannot remove completely the interference due to the semi orthogonality and limited dimensionality. In the second part of 
the paper, we propose a intelligent pilot assignment framework that reduces the remaining interference. We show that pilot assignment can be modeled as a max cut problem. We then propose an approximation algorithm, that optimizes the pilot allocation based only on the large scale fading coefficients.

Notations: The notations $A^{\dagger},\|A\|$ and $\operatorname{Tr}\{A\}$ are used for transpose, Euclidean norm and trace, respectively. We use $C N\left(0, \sigma^{2}\right)$ for a circular symmetric complex Gaussian distribution a with zero mean and variance $\sigma^{2}$.

\section{System Model ANd PREliminaries}

We consider a two-dimensional cellular network composed of $C$ hexagonal cells with one base station and $\mathrm{K}$ single omnidirectional antenna users in each cell. Each base station is equipped with $\mathrm{M}$ antennas . An example of the cells layout is given in FIGURE 1. We consider the case of frequency reuse factor of one where the same bandwidth is reused in all cells. The wireless channel from the $i^{t h}$ user in the $b^{t h}$ cell to the $j^{t h}$ BS is given by: $g_{i b}^{[j]}=\sqrt{\beta_{i b}^{[j]}} h_{i b}^{[j]}$. We assume that each channel coefficient can be decomposed as a product of the large-scale fading coefficient and the small-scale fading coefficient. The small-scale fading coefficients $h_{i b}^{[j n]}$ are modeled as Rayleigh fading, i.e., an i.i.d $C N(0,1)$ distributed random variables. The large-scale fading (LSF) coefficient $\beta_{i b}^{[j]} \in \mathbb{R}^{+}$depends on the distance between the user and BS and the shadowing. Typically, the distance between BS and a user is significantly larger than the distance between antennas, then the large-scale fading coefficient is the same for all the BS antennas. We suppose that the large-scale fading coefficient vary slower than small-scale fading coefficients which is particularly true when mobile users have low speed. We assume the block fading model for the small-scale fading coefficients, meaning they stay constant during small-scale coherent interval of $T$ symbols. We consider Time-Division Duplexing (TDD) mode where the entire frequency band is used for downlink and uplink transmissions by all base stations and all users. In this paper we concentrate on the uplink. Channel state information estimation is performed using orthonormal training sequences where in each cell the same set of $\tau$ training sequences $q_{i} \in \mathbb{C}^{\tau \times 1}\left(q_{i}^{\dagger} q_{j}=\delta_{i j}\right)$ is used with reuse factor of 1 .

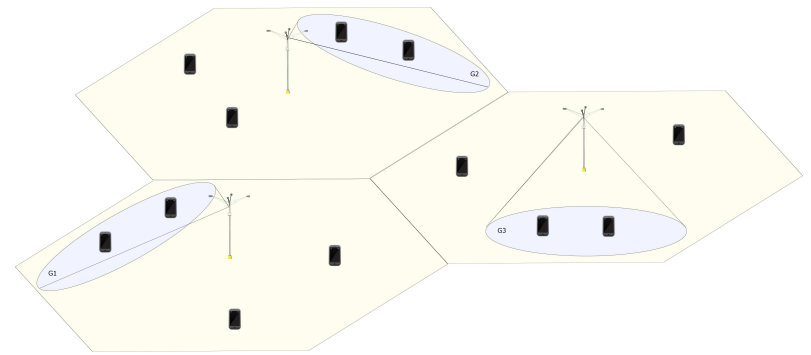

Fig. 1: Network Model

\section{GRoup BASED USER SCHEDULING}

In this section, we propose a user scheduling method that exploits the directions of user's channel [4] [2] without the need for substantial feedback. We first provide a general introduction to the proposed scheduling scheme and then, in the next subsection, we describe the scheduling algorithm in more details. As in [3][7], the key idea is to partition users into multiple groups so that each group has a distinguishable linear subspace. While in [3][7], the subspaces are defined by the dominant eigenvectors of the groups channel covariance matrix, we concentrate in this work on multiuser diversity and the users are grouped according to their channel directions. The grouping method used in this paper has the advantage to not require the estimation of user channel covariance matrix which may be cumbersome and quite complex especially in massive MIMO systems The clusters will be computed in each cell such that the signal of users in different clusters are centered in orthogonal subspaces. This enable us to effectively mitigate the pilot contamination effect and even reusing the same pilot sequence more than once in a given cell. Scheduling users based on the dominant sub-spaces of their signal, can reduce the mutual interference. The Key idea is to limit pilot contamination which is responsible for the persistent interference in the large antenna array regime. This will be done by allocating the same pilot sequence only to users in different clusters [7]. In order to exploit having multiple antennas at the BS without having full CSI, we propose a scheme in which the BS constructs random beams which will be used as downlink probes. The set of $M$ random beams $\phi=\left\{\phi_{1}, \phi_{2}, \ldots \phi_{M}\right\}$ with $\phi_{i}$ a random orthonormal vector $\in C^{M \times 1}$ is generated according to an isotropic distribution. Using this property we restrain the scheduled users to those having a predefined dominant subspace based on $d$ vectors of the set of the reference beams, where $d$ is the number of beams that are associated with each cluster. Using downlink probing simplifies the clustering procedure. While random beamforming has been already used in the literature [6],[4] for FDD systems with CSI feedback, we apply it here for downlink probing in TDD mode with uplink training. This enables a more dynamic system and mitigates the need for substantial feedback. In fact, this is quite advantageous since the resources needed for CSI acquisition scale up with the number of BS antennas in FDD systems. The number of different beams sets that are associated with the clusters is $G=\frac{M}{d}$. We will reuse the same reference beams in different cells. In the proposed setting, we consider a pilot reuse factor of 1 between the clusters in a given cell. Different pilot sequences will be used in neighboring cells which enables to reduce the pilot contamination effect thanks to pathloss. In fact, for a given user, copilot interference will be coming from distant or users with semi-orthogonal channels. Once the reference beams are allocated to the different cells, they will be transmitted so that the mobile users could determine if its channel is aligned enough with the reference beams corresponding to its serving cell. The detailed scheduling procedure is provided in the next subsection. 


\section{A. Training and Downlink Probing}

As in [6] [4], user selection will start by BSs sending their reference predefined beams which constitute a basis of the signal space. We suppose that user $l r$, ( $l^{\text {th }}$ user in cell $r$ ) knows $\frac{\left\|g_{l r}^{[r]^{\dagger}} \phi_{i}\right\|}{\left\|g_{l r}^{[r]}\right\|}$ for $i=1, \ldots, M$. This can be justified by measuring the strength of the signal on each beam given by $\left\|g_{l r}^{[r]^{\dagger}} \phi_{i}\right\|^{2}$ and an estimate of $\left\|g_{l r}^{[r]}\right\|^{2}$ which can be approximated by $M \beta_{l r}^{[r]}$. Assuming that we have $G$ different clusters, each one is associated with $d$ predefined reference beams $\Phi_{c}=\left[\phi_{i c}, i=1 \ldots d\right]$ for $c=1 \ldots G$. If the channel $g_{l r}^{[r]}$ of user $l r$ verifies:

$$
\frac{\left\|g_{l r}^{[r]^{\dagger}} \Phi_{c}\right\|}{\left\|g_{l r}^{[r]}\right\|}<\alpha, \text { for, } i=1 \ldots d, \text { for all but one } c \text { in }[1 \ldots G]
$$

meaning that user $l r$ will be affected to the cluster for which the normalized projection is greater than $\alpha$ since the user channel is well aligned with the reference beams associated with this cluster. Since users associated with different clusters have their channels centered in different subspaces, the same pilot sequences will be used with a reuse factor of 1 between the clusters. After downlink probing, the mobile users will feed back the index of their cluster to the BS which will conduct pilot assignment and select the users scheduled for transmission. In each group, a maximum of $\tau$ users will be scheduled, each one having a different pilot sequence. The main upside of using the downlink probing through reference beams, is to reduce the complexity of the user grouping scheme. In fact, in our setting we do not need to estimate the user channel covariance matrix (e.g. as in [3], [7]) which may be cumbersome and quite complex especially in massive MIMO systems. We are able to group users according to their channel subspaces dynamically through the use of reference beams and very limited feedback. In fact the users will only feed back an index. Note that $0<\alpha<1$ and can be defined dynamically as a function of the user density in the network. The same pilot sequences will be reused in all clusters within a single cell. In this case, we will have an interesting impact on the training phase since we can shorten the pilot transmission time. Supposing that, for each cell, we are going to schedule $K$ users and that we have $G_{c}$ clusters with $N_{b}$ direct neighboring cells. Then, in the classical MIMO setting with pilot aided channel estimation and universal pilot reuse, the training phase should at least have a duration of $K$ channel uses. In our clustering setting on the other hand, only $\frac{K\left(N_{b}+1\right)}{G_{c}}$ symbols are needed for training. By appropriately choosing $G_{c}$ and $d$, we are able to reduce the needed training resources for a given number of scheduled users. The copilot interference, in this case, is coming from users in different clusters or in distant cells. This will enable to reduce the impact of copilot interference.

\section{B. Group based Channel Estimation}

Once the users identify their serving cluster, the BS will select $\tau$ users per cluster to be scheduled for pilot transmission in order to estimate the effective CSI. In this section we introduce the index of the reference beams set to which, each user is assigned. The wireless channel from user $l$ in cell $r$ associated with cluster $j$ to BS $r$ is given by $g_{l r}^{[r j]}$. Here $\nu(b), \forall b=1 . . C$ gives the index of the beams groups allocated to cell $b$. Not that all the sets of beams can be used in each cell and the number of clusters $G_{c}$ can take values in $[1, G]$. $\chi(b)$ refers to the cells using the same set of pilot sequences as cell $b . q_{i}^{b}$ refers to the $i^{t h}$ pilot sequence used in cell $b$. Then during the training phase, the received pilot signal at BS $r$ taken here as a reference BS is given by:

$$
Y_{c}^{p}=\sum_{b=1}^{C} \sum_{j \in \nu(b)} \sum_{i=1}^{\tau} g_{i b}^{[r j]} q_{i}^{b^{\dagger}}+N_{p}
$$

Where $N_{p} \in \mathbb{C}^{M \times \frac{\tau\left(N_{b}+1\right)}{G_{c}}}$ is the additive white Gaussian noise matrix with i.i.d. $C N(0,1)$ entries. Conventionally, the Base Station correlates the training signal with the known pilot sequences of each user in order to obtain a channel estimate. Although we do not estimate the covariance matrix, we can use the information on the dominant subspace to further improve the estimation. Then the MMSE estimate of the $l^{\text {th }}$ user in cluster $k$ and cell $r$ is given by: $\hat{g}_{l r}^{[r k]}=\Theta_{l r}^{[r k]} \hat{g}_{l r}^{[r k]^{L S}}$ with $\hat{g}_{l r}^{[r k]^{L S}}$ being the least square estimate of the channel given by: $\hat{g}_{l r}^{[r k]^{L S}}=Y_{c}^{p} \times q_{l}^{r}=\sum_{b \in \chi(r)}^{C} \sum_{j \in \nu(b)} g_{l b}^{[r j]}+N_{p} q_{l}^{r}$ and $\Theta_{l r}^{[r k]}=R_{l r}^{[r k]}\left(I+\sum_{b \in \chi(r)}^{C} \sum_{j \in \nu(b)} R_{l b}^{[r j]}\right)^{-1}$.

Since $\left\{\phi_{1}, \phi_{2}, \ldots \phi_{M}\right\}$ is an orthonormal basis of the channel, then $g_{l b}^{[r k]}$ can be written as $g_{l b}^{[r k]}=\sqrt{\beta_{l r}^{[r k]}} \sum_{i=1}^{M} a_{i l r}^{[r k]} \phi_{i}$. Note that $R_{l r}^{[r k]}$ the covariance matrix of the channel of user $l, r$. The Mean square error of channel estimation is given in our case by:

$$
\begin{aligned}
M S E_{l r} & =\mathbb{E}\left[\left\|g_{l r}^{[r k]}-\hat{g}_{l r}^{[r k]}\right\|^{2}\right] \\
& =\operatorname{Tr}\left\{R_{l r}^{[r k]}-R_{l r}^{[r k]^{2}}\left(I+\sum_{b \in \chi(r)}^{C} \sum_{j \in \nu(b)} R_{l b}^{[r j]}\right)^{-1}\right\}
\end{aligned}
$$

Then the channel estimate of user $l, r$ can be written as:

$$
\begin{aligned}
\hat{g}_{l r}^{[r k]} & =R_{l r}^{[r k]}\left(I+\sum_{b \in \chi(r)}^{C} \sum_{j \in \nu(b)} R_{l b}^{[r j]}\right)^{-1} \hat{g}_{l r}^{[r k]^{L S}} \\
& =\Phi A_{l r}^{[r k]} \Phi^{\dagger} \hat{g}_{l r}^{[r k]^{L S}}
\end{aligned}
$$

where $A_{l r}^{[r k]}$ is a diagonal matrix with $\left(A_{l r}^{[r k]}\right)_{i i}=$ $\frac{\beta_{l r}^{[r k]}}{1+\sum_{b \in \chi(r)}^{C} \sum_{j \in \nu(b)} \beta_{l b}^{[r j]}}$ Since we enforce semi-orthogonality among users in different clusters before the training phase, copilot users within a given cell will have their signals confined in different subspaces each one with dimension $d$. We consider 
that $\Phi_{k}=\left\{\phi_{(k-1) d+1} \ldots \phi_{k d}\right\}$ are the reference beams vectors associated with cluster $k$. With $\alpha \longrightarrow 0$, we reduce the interference between the groups since we confine more and more the channels to defined subspaces of rank $d$. With $\alpha \longrightarrow 0$ we have $\left|a_{i l r}^{[r k]^{2}}\right|^{2} \longrightarrow 0$ for $\forall i>(k-1) d+1$ and $i<(k d)$.

Therefore with $\alpha \approx 0, g_{l r}^{[r k]} \in \operatorname{Span}\left\{\phi_{(k-1) d+1} \ldots \phi_{k d}\right\}$ and the rest of copilot users is either confined in orthogonal subspace or coming from distant cells. The resulting channel estimate is given by:

$$
\begin{aligned}
\lim _{\alpha \rightarrow 0} \Phi_{k}^{\dagger} \hat{g}_{l r}^{[r k]}= & \Phi_{k}^{\dagger} R_{l r}^{[r k]}\left(I+\sum_{\substack{b \in \chi(r) \\
b \neq r}} \sum_{j \in \nu(b)} R_{l b}^{[r j]}\right)^{-1} \\
& \left(\sum_{\substack{b \in \chi(r) \\
b \neq r}} \sum_{j \in \nu(b)} g_{l b}^{[r j]}+N_{p} q_{l}^{r}\right)
\end{aligned}
$$

Having a lower $\alpha$ enables us to have a better channel estimate reducing the interference and enhancing the spectral efficiency. Note that with $\alpha=0$, the only left copilot interference comes from distant cells in which the same set of pilots is reused. In our setting, thanks to MMSE channel estimation, we are able to separate copilot users signals since each is centered in a different subspace. This enables us to reduce the pilot contamination problem. We propose that the pilot reuse factor would be 1 between the groups meaning that the same pilot sequence can be used $G_{c}$ times within one cell which enables us to reduce the training duration compared with a classical system for the same number of scheduled users. Since copilot signals are centered in different subspaces, increasing the reuse of a given training sequence will not degrade the achievable spectral efficiency of the system.

\section{SPECTRAL EFFICIENCY IMPROVEMENT}

In this section we investigate the spectral efficiency of our system under direction based user scheduling. We derive SINR expressions for uplink when the number of antennas goes to infinity. Upon receiving the uplink signal the BS will use the MMSE estimate of the users channels. The decoding in MIMO systems can be done through, Zeroforcing, MMSE, or Matched filtering receivers. In this paper we use the Matched filtering approach at the reception. Using MMSE channel estimation, we consider $\tilde{g}_{l r}^{[r k]}$ to be the error in channel estimation, $\tilde{g}_{l r}^{[r k]}=g_{l r}^{[r k]}-\hat{g}_{l r}^{[r k]}$. In order to complete our analysis we need the following properties of $\hat{g}_{l r}^{[r k]}$ and $\tilde{g}_{l r}^{[r k]}$ which can easily be derived from their definitions: $\hat{g}_{l r}^{[r k]} \sim C N\left(0, C_{l r}^{[r k]}\right)$ and $\tilde{g}_{l r}^{[r k]} \sim C N\left(0, \tilde{C}_{l r}^{[r k]}\right)$ with $\tilde{C}_{l r}^{[r k]}=R_{l r}^{[r k]}-C_{l r}^{[r k]}$. Note that $\hat{g}_{l r}^{[r k]}$ and $\tilde{g}_{i b}^{[r j]}$ are uncorrelated for all indexes $r, l, j, i, b$. and $C_{l r}^{[r k]}$ is a diagonal matrix with: $\left(C_{l r}^{[r k]}\right)_{i i}=\frac{\beta_{l r}^{[r k]^{2}}}{1+\sum_{b \in \chi(r)}^{C} \sum_{j \in \nu(b)} \beta_{l b}^{[r j]}}$ We concentrate on cell $r$ taken as a reference. After the training phase and upon receiving the uplink signal, the BS applies a matched filter using $\hat{g}_{l r}^{[r k]^{\dagger}}$ in order to detect the data from user $(k, l, r)$.
Then the expected value of $d_{k l r}$ is given by:

$$
\hat{d}_{k l r}=\sum_{b=1}^{C} \sum_{j \in \nu(b)} \sum_{i=1}^{\tau} \hat{g}_{l r}^{[r k]^{\dagger}} g_{i b}^{[r j]} d_{j i b}+\hat{g}_{l r}^{[r k]^{\dagger}} N_{u}
$$

where $d_{j i b}$ is the data symbol of user $j, i, b$ with $\mathbb{E}\left(\left|d_{j i b}\right|^{2}\right)=$ 1. We see that with $\alpha \approx 0$ we completely remove the interference due to pilot contamination coming from different user clusters and the only remaining copilot contamination comes from distant cells in which the same training sequences are used. But another component of the interference becomes dominant. In fact, in classical Random Beam-forming settings, [6] [4], only one user is scheduled according to a given criterion from the set of users associated with a given Beam. In our setting $\tau$ users from each cluster are scheduled. These users have channels that are to some degree correlated. Then even when using orthogonal pilot sequences, it is difficult to separate their signals. In order to reduce this effect we tend to have $d \longrightarrow \infty$ in the infinite antenna regime or at least a large number of beams $d$ which reduces the correlation between the channels of users within the same group. When $d$ is large, users in the same cluster will have channels distinct enough so that the detection of their signals is possible. When the number of antennas is large, interference not related to pilot contamination vanishes. Considering a constant number of clusters $G$ and $d$ very large we obtain the following result:

Lemma 1. With a matched filter receiver $\left(\Phi_{k}^{\dagger} \hat{g}_{l r}^{[r k]}\right)^{\dagger}$ and large $d$ the uplink SINR of user $l, r, k$ is lower bounded by:

$$
S I N R_{l r}^{[k]}=\frac{\beta_{l r}^{[r k]^{2}}}{\sum_{\substack{b \in \chi(r) \\ b \neq r}} \sum_{j \in \nu(b)} \beta_{l b}^{[r j]^{2}}+\sum_{\substack{j \in \nu(r) \\ j \neq k}} \beta_{l r}^{[r j]^{2}} \xi}
$$$$
\text { with } \xi=\alpha^{4}
$$

Proof: The detailed proof can be found in [12]

The first term in the denominator represents the interference coming from users using the same pilot sequences in distant cells where the second term is the interference coming from clusters using different beams within cell $r$. Having a lower $\alpha$ will result in better spectral efficiency. When $\alpha \longrightarrow 0$ the persistent interference will vanish, leaving only a weak component coming from distant cells. While it is very tempting to have a vanishing interference in the asymptotic regime, $\alpha$ can not be made low without having impact on the system. In fact in addition to spectral efficiency, $\alpha$ has an impact on the probability of having a user with the desired channel direction.

\section{Pilot Assignment Using Max Cut}

The proposed dynamic clustering scheme enables to improve the spectral efficiency but we are not able to completely remove copilot interference. In fact $\alpha$ is typically different from 0 and the limited training resources means that the same pilot sequences need to be reused in distant cells. 


\section{A. Problem Formulation}

In order to further reduce copilot interference, we propose an intelligent pilot assignment method. In fact, the SINR in the large antenna regime can be enhanced if the users are associated with their pilot sequences such that copilot interference is minimized. After downlink probing, the users are separated into clusters (as explained in section III.A). The Base station selects then at most $\tau$ users per cluster (since the number of users in each cluster can be higher than $\tau$ ) to be active during one coherence time. This user selection can be done using any known scheduling policy. Then the network will assign efficiently the training sequences to the scheduled users in such a way to reduce the copilot interference. In this section, we focus on this pilot assignment problem. The basic idea is to infer the interference from the Large Scale Fading coefficients that depend on the mobile user geographical location. The aim is to maximize the sum rate by efficiently assigning the pilot sequences. We construct the following combinatorial optimization problem :

$$
\begin{gathered}
\max _{X} \sum_{b=1}^{C} \sum_{j \in \nu(b)} \sum_{i=1}^{\tau} \sum_{p=1}^{\tau} \log \left(1+\frac{\beta_{i b}^{\prime[r j]^{2}} x_{i b}^{[j p]}}{\sum_{s \in \chi(b)}^{C} \sum_{u \in \nu(s)} \sum_{n=1}^{\tau} \beta_{n s}^{\prime[r u]^{2}} x_{n s}^{[u p]}}\right) \\
\text { s.t } \sum_{p=1}^{\tau} x_{i b}^{[j p]} \leq 1 \quad \forall b=[1 . . C], \forall i=[1 . . \tau], \forall j \in \nu(b) \\
\sum_{i=1}^{\tau} x_{i b}^{[j p]} \leq 1 \quad \forall p=[1 . . \tau], \forall b=[1 . . C], \forall j \in \nu(b)
\end{gathered}
$$

With $x_{i b}^{[j p]}= \begin{cases}1 & \text { if user (i,b,j) uses pilot } \mathrm{p} \\ 0 & \text { otherwise }\end{cases}$

Here user $(i, b, j)$ means user $i$ in cell $b$ and cluster $j$ and $p$ gives the index of the used pilot sequence. $\beta_{i b}^{\prime[r j]^{2}}$ is equal to $\beta_{i b}^{[r j]^{2}} \xi$ or $\beta_{i b}^{[r j]^{2}}$ depending on whether the interference is impacting users from the same cell or from distant ones with the same pilot sequences. The first constraint guarantees that a given user is allocated only one training sequences. The second constraints guarantees that a given pilot sequence is used only once in each cluster. Finding an optimal solution for the proposed optimization problem is computationally prohibitive. So in order to solve this problem we propose a graphical approach with an approximation algorithm that is guaranteed to achieve at least a constant relative performance guarantee. In order to optimize the pilot assignment we propose to construct a graph representing the mobile users and the mutual interference based on the large scale fading coefficients. To do this, we need first to proceed by simplifying the objective function of the optimization problem. Once the pilots are assigned the sum rate is given by:

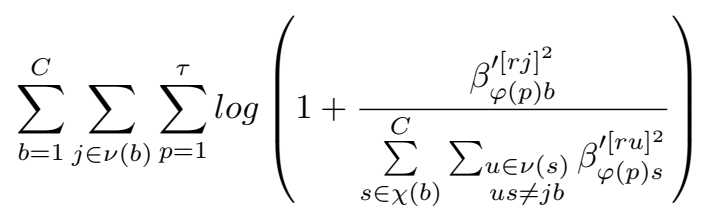

Here $\varphi(p)$ gives the index of the user using pilot sequence $p$ in each cell and cluster. Assuming high SINR, we obtain the following expression equivalent to the sum rate:

$$
\sum_{b=1}^{C} \sum_{j \in \nu(b)} \sum_{p=1}^{\tau} \log \left(\beta_{\varphi(p) b}^{\prime[r j]^{2}}\right)-\log \left(\sum_{s \in \chi(b)}^{C} \sum_{\substack{u \in \nu(s) \\ u s \neq j b}} \beta_{\varphi(p) s}^{\left[[r u]^{2}\right.}\right)
$$

We can further simplify this expression by using the results from [11], replacing the arithmetic mean with geometric mean. Then $\forall i$ we can write: $\left(\sum_{s \in \chi(b)}^{C} \sum_{\substack{u \in \nu(s) \\ u s \neq j b}} \beta_{\varphi(p) s}^{\prime[r u]^{2}}\right) \approx$ $\left(|\chi(b)| G_{c}-1\right)^{|\chi(b)| G_{c}}\left(\prod_{\substack{s \in \chi(b) u \in \nu(s) \\ u s \neq j b}} \beta_{\varphi(p) s}^{\prime[r u]^{2}}\right)^{\frac{1}{\left(|\chi(b)| G_{c}-1\right)}}+U$. Here $U$ is due to the difference between the arithmetic and geometric means. $U$ depends on the smallest and largest values in addition to the variance of the LSF coefficients. This approximation becomes accurate when the variance of the values of LSF coefficients is small. Since the persistent interference coming from co-pilot users in different neighboring clusters is attenuated by the semi orthogonality and the remaining interference is from distant cells, the variation of the LSF is small making the above approximation efficient. Then the aim of the pilot assignment optimization is to maximize the following expression:

$$
\sum_{b=1}^{C} \sum_{j \in \nu(b)} \sum_{p=1}^{\tau} \log \left(\beta_{\varphi(p) b}^{\prime[r j]^{2}}\right)-\log \left(\prod_{\substack{s \in \chi(b)) \\ u \in \nu(s) \\ u \neq j b}} \beta_{\varphi(p) s}^{[r r u]^{2}}\right)
$$

Since the users are already selected for pilot transmission, the first term of the sum does not play any role in the optimization. Finally the original problem is equivalent to minimizing:

$$
\begin{array}{r}
\min _{X} \sum_{\substack{b=1 \\
s \in \chi(b)}} \sum_{\substack{j \in \nu(b) \\
u \in \nu(s)}} \sum_{i=1}^{\tau} \sum_{p=1}^{\tau}\left(\log \left(\beta_{i s}^{\prime[r u]^{2}}\right)+\log \left(\beta_{n b}^{\prime[r j]^{2}}\right)\right) x_{i s}^{[u p]} x_{n b}^{[j p]} \\
\text { s.t } \sum_{p=1}^{\tau} x_{i b}^{[j p]} \leq 1 \quad \forall b=[1 . . C], \forall i=[1 . . \tau], \forall j \in \nu(b) \\
\sum_{i=1}^{\tau} x_{i b}^{[j p]} \leq 1 \quad \forall p=[1 . . \tau], \forall b=[1 . . C], \forall j \in \nu(b)
\end{array}
$$

Using the mentioned simplifications, we are now able to construct the graphical framework for the problem of pilot allocation.

\section{B. Graphical Modeling and Pilot allocation}

We construct corresponding undirected graph for the pilot allocation problem $\mathbb{G}=(V, E)$ where $V$ represent the users and each edge from $E$ will be associated with a weight that 
characterizes the mutual interference between the users in the uplink based on the large scale fading. Note that $\mathbb{G}=(V, E)$ will be given by $N_{b}+1$ disjoint graphs each one associated with a set of pilot sequences. Figure 2 shows the interference graph for a given set of $\tau$ pilot sequences. We specify the

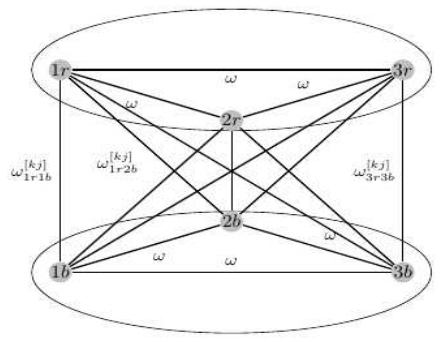

Fig. 2: Interference graph

edge's weight so that we approach the effective interference between the users in the uplink. In order to make sure that a given pilot sequence is allocated only once in each cluster, the weight of the links between users in the same cluster will be given a large value $\omega$. The weight of the link between two users $k, l, r$ and $j, i, b$ in different cells is given by: $\omega_{l r, i b}^{[k j]}=\log \left(\beta_{l r}^{\prime[r k]^{2}}\right)+\log \left(\beta_{i b}^{\prime[r j]^{2}}\right)$. The optimization problem aims at putting users that interfere strongly in different pilot groups, resulting in the reduction of the copilot interference. Putting two users $k, l, r$ and $j, i, b$ in the same group $p$ means that $x_{i b}^{[j p]}=1$ and $x_{l r}^{[k p]}=1$. Then given the pilot allocation graph the aim is to partition the graph into $\tau$ disjoint Clusters $\left[P_{1} \ldots P_{\tau}\right]$ such that $\cup_{i=1}^{\tau} P_{i}=V$ and $\sum_{i=1}^{\tau} \sum_{a, b \in P_{i}} \omega_{a b}$ is minimized. This means that edges where the interference is high are removed since the corresponding users will be allocated different pilot sequences. Recall that the weight of the edges between users in the same cluster will be given a large value $\omega$. This will guarantee that each pilot sequence is used only once in each cluster. After building the Pilot association graph, we can notice that our optimization problem is equivalent to the $M A X-\tau-C U T$ problem for $N_{b}+1$ disjoint graphs. In graph theory the $M A X-\tau-C U T$ problem aims at partitioning the vertices's of the graph so that the weight of the edges crossing the cut is maximized. In our setting we partition the users into $\tau\left(N_{b}+1\right)$ groups, each one using one of the orthogonal pilot sequences. Solving the $M A X-\tau-C U T$ problem for each set of $\tau$ pilot sequences results in the minimization of the edges sum weight within each group, meaning a minimization of the mutual interference in each copilot users group. In this section we use an approximation algorithm to solve the pilot association problem. It is known that, $M A X-\tau-C U T$ problem is NP-hard. Which means that the optimal solution for this problem is computationally prohibitive. Then we apply the approximation algorithm proposed in [8] which produces an approximate solution that attains, at least, $\left(1-\frac{1}{\tau}\right)$ of the optimal solution of the $\max -\tau$-cut problem. The proposed algorithm can produce a solution in which the inter-cluster weight is at least $\left(1-\frac{1}{\tau}\right)$ times the optimal sum weight for each set of cells using the same pilot sequences. Since the edge's weight represents the mutual interference that results from sharing a pilot sequence, then the max cut algorithm will assign different pilot sequences for users that interfere strongly. The algorithm proceeds by assigning users to the clusters such that, we minimize the intra-cluster interference at each step. The detailed description of the algorithm is given in the next table. The complexity of the proposed algorithm is proportional to the number of edges, nodes and clusters. The proposed method enables to efficiently allocate pilot sequences based only on large scale fading coefficient which are slowly varying and easily estimated compared with the instantaneous CSI.

Pilot assignment algorithm

For each set of cells using the same pilot sequences:

Initialize:

intra-cluster interference $W_{i}=0, \forall i=1 . . \tau$

1. Assign the $\tau$ users in cell 1 to different clusters

2. Randomly Order the rest of the users

3 . Take the next Node $l$ and assign it to group $i^{*}$ for which the sum $W_{i}^{l}$ is minimized where $W_{i}^{l}=\sum_{a \in P_{i}} \omega_{l a}$

4. Update the intragroup interference of group $i^{*}$ such that $W_{i^{*}}=W_{i^{*}}+W_{i^{*}}^{l}$

5. Repeat steps 3-4 until all nodes are assigned.

\section{NUMERICAL RESULTS}

In this section we provide some numerical results demonstrating the performance of the proposed user clustering scheme and the pilot assignment approach. We consider an hexagonal cell network with 7 cells of $1 \mathrm{Km}$ radius. The mobile users are located uniformly at random in each cell and we assume that no user is closer to its serving BS than $r_{0}=10 \mathrm{~m}$. For large scale fading coefficients we take into consideration only path-loss, where $\beta_{i b}^{\prime[r u]}=\left(R_{i b}^{[r]}\right)^{(-\sigma)}$, between the $i^{t h}$ user in the $b^{\text {th }}$ cell and the $j^{t h} \mathrm{BS}$, where the path-loss exponent $\sigma=3$. We select the parameter $\alpha$ from the interval $[0,1]$. We consider a coherence interval of $T=200$ symbols split between training and data transmission. The spectral efficiency is measured in bits/second/Hz. Figure 3 shows a comparison in mean square error in channel estimation for a reference user. We can see that the MSE is an increasing function of $\alpha$. We notice that for low values of $\alpha$ we have a considerably enhanced channel estimate, since low $\alpha$ yields almost orthogonal copilot signal. Figure 4 shows a comparison of the CDFs of achievable sum rates. We notice the considerable improvement due to user clustering. Here we considered the large antenna regime where $d=10$. For 7 cells containing each one cluster of users, we achieve a total sum capacity (all users in the network) up to $289 \mathrm{bit} / \mathrm{s} / \mathrm{hz}$ with probability 0.8 with the grouping scheme while a classical user scheduling achieves a sum capacity up to $140 \mathrm{bit} / \mathrm{s} / \mathrm{hz}$ with the same probability. This improvement is due to the reduction of the persistent interference in massive MIMO systems due to pilot contamination since copilot signals are semi-orthogonal with user clustering. Figure 5 shows a comparison of the CDFs 


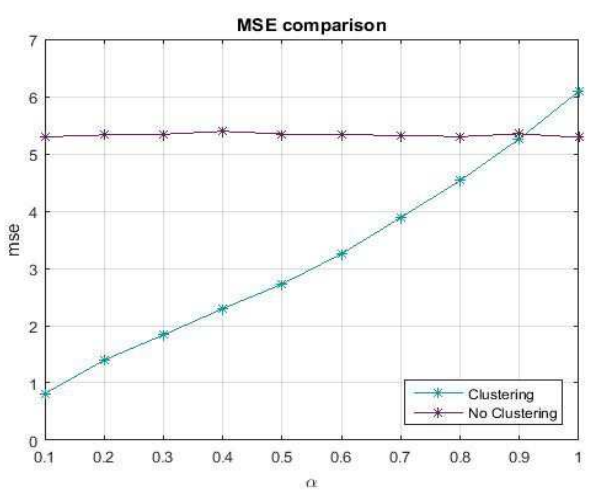

Fig. 3: Mean square Error in reference user channel estimation

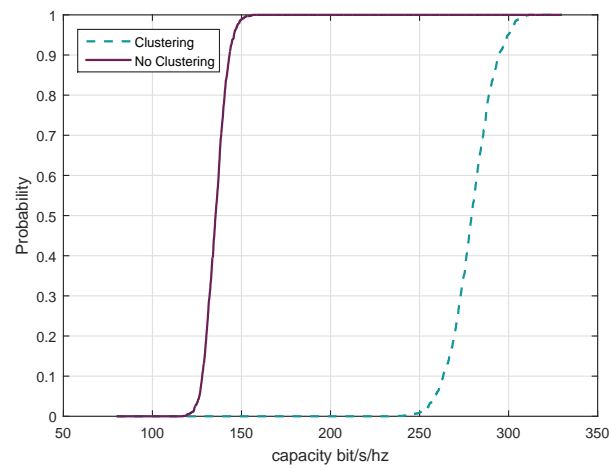

Fig. 4: CDF Sum Capacity comparison

of achievable sum rates when applying the proposed pilot assignment algorithm. Although the curves seem to be close to each other, the improvement is not negligible. In fact, with the proposed pilot assignment method we achieve a sum capacity up to $291.6 \mathrm{bit} / \mathrm{s} / \mathrm{hz}$ with probability 0.8 while a random pilot allocation achieves a sum capacity up to $289 \mathrm{bit} / \mathrm{s} / \mathrm{hz}$ with the same probability. This means that the proposed approximation algorithm results in a gain of approximately $52 \mathrm{Mbits} / \mathrm{s}$ for a bandwidth of $20 \mathrm{Mhz}$. It is worth mentioning that by using our proposed direction based scheduling, we already reduced the interference considerably and the copilot interference comes from users in distant clusters. Therefore, one cannot expect a much larger gain from the pilot assignment framework.

\section{CONCLUSION}

In this paper we studied the channel direction based user scheduling in order to mitigate pilot contamination in a massive MIMO system. We proposed a new scheduling approach for TDD systems. Downlink probing is used, reducing the complexity of the scheduling and the need for substantial feedback. The proposed scheme enables a more aggressive reuse of the pilot sequences without degrading the performance. Results show that this grouping improves the spectral efficiency and channel estimation accuracy. Since the proposed grouping approach cannot remove completely the interference,

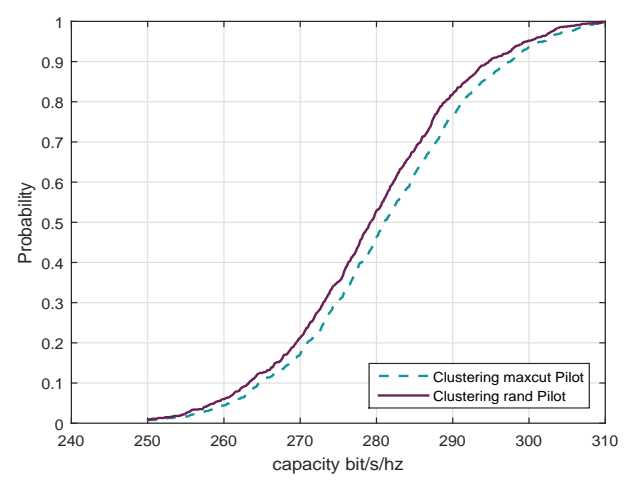

Fig. 5: CDF Sum Capacity comparison Pilot Assignment

we also proposed a graphical framework for pilot assignment in order to further improve the performances of the system. We proved that pilot assignment can be approximated by a max cut problem in the high SINR regime. We then provide an approximation algorithm to optimize the pilot allocation.

\section{REFERENCES}

[1] T. L. Marzetta, "Noncooperative cellular wireless with unlimited numbers of base station antennas," IEEE Transactions on Wireless Communications, vol. 9, no. 11, pp. 3590-3600, November 2010.

[2] H. Yin, D. Gesbert, M. Filippou and Y. Liu, "A Coordinated Approach to Channel Estimation in Large-Scale Multiple-Antenna Systems," IEEE Journal on selected areas in communications, vol 31, no. 2, pp. 264 - 273, February 2013.

[3] J. Nam, A. Adhikary, J. Y. Ahn and G. Caire, "Joint Spatial Division and Multiplexing: Opportunistic Beamforming, User Grouping and Simplified Downlink Scheduling," IEEE Journal of Selected Topics in Signal Processing, vol 8,no. 5, pp. 876 - 890, Mars 2014.

[4] G. Lee, Y. Sung, "A New Approach to User Scheduling in Massive Multi-user MIMO Broadcast Channels,"arXiv:1403.6931

[5] Adhikary, Ansuman, Ebrahim Al Safadi, Mathew K. Samimi, Rui Wang, Giuseppe Caire, Theodore S. Rappaport, and Andreas F. Molisch, "Joint spatial division and multiplexing for mm-wave channels," arXiv preprint arXiv:1312.2045, 2014.

[6] M. Sharif and B. Hassibi, "On the capacity of MIMO broadcast channels with partial side information," IEEE Trans. Inf Theory, vol. 51, no. 2, pp. 506 -522, Feb. 2005

[7] Junyoung Nam, Giuseppe Caire, Young-Jo Ko, Jeongseok Ha, "On the Role of Transmit Correlation Diversity in Multiuser MIMO Systems," submitted to IEEE Trans. Inf Theory.

[8] S. Sahni and T. Gonzalez, "P-complete approximation problems," Journal of the Association for Computing Machinery, vol.23, No.3, pp.555-565, July 1976. 
[9] Saeid Haghighatshoar, Giuseppe Caire, "Channel Vector Subspace Estimation from Low-Dimensional Projections," arXiv preprint arXiv:1509.07469, 2015.

[10] R. Y. Chang, Z. Tao, J. Zhang, C. C. J. Kuo, "Multicell OFDMA Downlink Resource Allocation Using a Graphic Framework," IEEE Trans. on Vehicular Technology, Vol.58, no.7, pp. 3494 - 3507, Sep.2009.

[11] S. H. Tung, "On lower and Upper Bounds of the Difference Between the Arithmetic and the Geometric Mean," Mathematics of Computation, Vol. 29, no. 131, pp. 834836, July 1975.

[12] S. Hajri, M. Assaad, and G. Caire, "Scheduling in Massive MIMO: User clustering and pilot assignment," extended version, available online at http://www.12s.centralesupelec.fr/perso/salaheddine.hajri 